\title{
O desenvolvimento da avaliação psicológica no Brasil: Avanços históricos e desafios
}

\author{
Solange Muglia Wechsler ${ }^{1}$ \\ Pontificia Universidade Católica de Campinas, Campinas-SP, Brasil \\ Claudio Simon Hutz \\ Universidade Federal do Rio Grande do Sul, Porto Alegre-RS, Brasil \\ Ricardo Primi \\ Universidade São Francisco, Campinas-SP, Brasil
}

\section{RESUMO}

A história da avaliação psicológica no Brasil é encontrada de maneira dispersa, existindo a necessidade de congregar essa informação, assim como os marcos da sua evolução. O seu desenvolvimento se torna mais nítido a partir dos últimos 20 anos, com a fundação do Instituto Brasileiro de Avaliação Psicológica e do apoio ao movimento pelo Conselho Federal de Psicologia ao organizar as comissões de avaliação psicológica e o Sistema de Avaliação dos Testes Psicológicos-SATEPSI. Os resultados podem ser percebidos com o aumento sensível de laboratórios para criação e validação dos testes em diferentes pontos do país, grupos de pesquisa, criação de associações e publicações na área. Pode-se concluir que a avaliação psicológica no Brasil atingiu um considerável nível de maturidade, podendo ser considerada como um exemplo para outras nações da América do Sul.

Palavras-chave: avaliação psicológica; testes psicológicos; validade; história teste; cultura.

\section{ABSTRACT - The development of psychological assessment in Brazil: Historical advances and challenges}

The history of psychological assessment in Brazil is found in a scattered way, so there is the need to gather this information as well as to present the milestones of its evolution. Undoubtedly, this evolution has been noticed since the last 20 years, with the foundation of the Brazilian Institute of Psychological Assessment-IBAP and the support from the Federal Council of Psychology which organized commissions for psychological assessment and created the System for Evaluating Psychological Tests-SATEPSI. The results can be observed with the increase of laboratories for test construction and validation, research groups, professional associations as well as publications. We conclude that psychological assessment in Brazil reached a considerable level of maturity and can be considered as an example for other South American countries.

Keywords: Psychological assessment; psychological tests; validity; test movement; culture.

\section{RESUMEN - El desarrollo de la evaluación psicológica en Brasil: Avanzos históricos y retos}

La historia de la evaluación psicológica en Brasil se encuentra de manera dispersa y existe a necesidad de reunir esta información y indicar los hitos de su evolución. Sin duda este desarrollo se vuelve más nítido a partir de los últimos 20 años, con la fundación del Instituto Brasileño de Evaluación Psicológica, y del apoyo al movimiento por el Consejo Federal de Psicología organizando las comisiones de evaluación psicológica y el Sistema de Evaluación de las Pruebas Psicológicas- SATEPSI. Los resultados pueden ser percibidos con el aumento sensible de laboratorios para crear y validar pruebas psicológicas en diferentes puntos del país, grupos de investigación, creación de asociaciones y publicaciones en el área. Se puede concluir que la evaluación psicológica en Brasil alcanzó un considerable nivel de madurez, pudiendo ser considerada como un ejemplo para otras naciones de América del Sur.

Palabras claves: Evaluación psicológica; pruebas psicológicas; validez; testes psicológicos; cultura.

A avaliação psicológica é umas das áreas mais importantes da Psicologia pois possibilita a compreensão do funcionamento psíquico e comportamental do indivíduo (American Educational Research, American Psychological Association, National Council of Measurement in Education, 2014). Os testes psicológicos representam uma contribuição essencial para esta área na medida em que permitem o diagnóstico e a intervenção em diferentes contextos, sendo utilizado em nível universal para todas as faixas etárias (Byrne et al., 2009; Ever et al., 2013; Geinsiger 2013). Diretrizes internacionais, como por exemplo da International Test Commission (2018), têm sido divulgadas no sentido de orientar pesquisadores e profissionais sobre os critérios científicos na prática e nas investigações no uso de testes psicológicos, sejam estes em forma impressa ou digital.

Endereço para correspondência: Pontifícia Universidade Católica de Campinas, Curso de Pós-graduação em Psicologia do Centro de Ciências da Vida. Avenida John Boyd Dunlop, s/n, Jardim Ipaussurama, 13060-904, Campinas, SP.E-mails: wechsler@lexxa.com.br; wechsler@puc-campinas.edu.br Agradecimentos: Os autores agradecem as valiosas contribuições sobre a história do movimento prestadas pelo Dr. Luiz Pasquali, da Universidade de Brasília. 
O desenvolvimento da área de testes psicológicos em um país depende, entretanto, de várias circunstâncias, segundo Oakland (2009), tais como a existência de uma atitude positiva para sua utilização, o avanço da disciplina de Psicologia, a qualidade da formação, a existência de associações profissionais fortes para sua regulamentação, a universalidade da educação e a existência de mercado para testes. Traçando um paralelo com nossa situação, podemos considerar que o movimento da avaliação psicológica no Brasil passou por distintas fases que refletem o nosso crescimento e desafios durante diferentes períodos (Pasquali, 2016; Hutz \& Primi, 2006; Noronha \& Reppold, 2010; Wechsler, 2013), tendo alcançado alguns dos pressupostos exigidos para considerarmos que esta área conseguiu atingir consideráveis níveis de maturidade e qualidade cientifica nos últimos 20 anos, como destacaremos a seguir.

\section{Panorama Histórico: $O$ interesse pela avaliação psicológica e educacional}

Certamente o movimento mundial na área da avaliação psicológica, em meados do século XIX, teve impacto na história brasileira. No âmbito internacional, devem ser destacados os nomes de Wundt, que criou o primeiro laboratório experimental na Alemanha, em 1862, para medir os processos mentais através da introspecção, de Francis Galton, em 1884, que organizou o primeiro laboratório com medidas antropométricas para avaliar os as funções mentais na International Health Exibiton in Londres. Deve também ser ressaltado que o termo "testes mentais" foi cunhado por James MacKeen Cattell, em 1890, para anunciar a sua bateria de testes, inspirada em Wundt e Galton (Flanagan, \& McDonough, 2018; Gregory, 2015; Kaplan \& Sacuzzo, 2013).

Os primeiros esforços para medir diferenças individuais eram direcionadas para avaliar as medidas fisiológicas, tais como tempo de reação e sensação de dor. Entretanto, deve-se a Alfred Binet, em 1895, a grande contribuição de construir medidas para avaliar os processos mentais, culminando com o primeiro teste para avaliar a inteligência infantil, com a colaboração de HenriSimon, denominado Teste de Binet-Simon (Satler, 2008). Atribui-se a Binet a influência sobre o médico Manuel Bonfim, que criou, no Rio de Janeiro, com a ajuda de Joaquim Medeiros, o primeiro serviço de psicologia aplicada, também denominado Laboratório Pedagogium, em 1890 (Gomes, 2004; Portugal, 2010);

No início do século XX, encontramos dois centros de psicologia aplicada (conhecidos como laboratórios) em São Paulo, fundados um em 1906 e outro em 1914, que se dedicavam, principalmente, às pesquisas sobre aprendizagem, utilizando testes de desenvolvimento mental e de aprendizagem para leitura e escrita (Pasquali, 2016). A organização do primeiro centro é creditada à Clemente Quaqlio, estando sediado em um grupo escolar na cidade de Amparo (SP). Por sua vez, o psicólogo italiano Ugo Pizzoli em 1914, também reorganizou e ampliou o centro de psicologia da Praça da República, que foi posteriormente absorvido pela Universidade de São Paulo, após sua fundação em 1934 (Pfromm Netto, 1981; Wechsler, 2001).

Em outras regiões do Brasil, em torno de 1920, também aconteciam importantes momentos na história da avaliação psicológica. No Recife, destaca-se a contribuição de Ulysses Pernambuco, que fundou o Instituto de Seleção e Orientação Profissional, posteriormente conhecido como Instituto de Psicologia (Antunes, 2002). Já em 1924 encontramos a primeira adaptação da escala Binet-Simon para o Brasil por Isaias Alves (Pasquali, 2001). Também em Minas Gerais, ressalta-se a contribuição da notável psicóloga russa Helena Antipoff, que organizou um programa na Fazenda do Rosário para atender crianças excepcionais, consideradas como sendo aquelas que tinham dificuldades de adaptação nas escolas, utilizando os testes de inteligência do Binet além de fichas comportamentais para classificar o seu nível intelectual, culminando na fundação da Sociedade Pestalozzi em 1932 (Rafante \& Lopes, 2008).

\section{Os Primeiros Testes Brasileiros}

A influência do movimento internacional foi decisiva no interesse em publicação de livros e testes brasileiros. As conferências de Henri Pierón na sua vinda ao Rio de Janeiro em 1921 e a publicação do livro "Tests", escrito por José Joaquim Medeiros e Albuquerque em 1924, podem ser considerados com marcos de influência para a área (Gomes, 2004). Também se ressalta nesta época as publicações dos livros "O método de testes" por Manuel Bonfim, em 1931, e de Alves em 1932 "Testes de inteligência nas escolas" (Monarcha, 2008).

Atribui-se a Lourenço Filho a criação do primeiro teste brasileiro "Teste ABC de Prontidão Escolar" em 1933, que visava produzir um diagnóstico da maturidade psicológica do aluno para a aprendizagem, sendo composto por oito subtestes: coordenação viso-motora, memória imediata, memória motora, memória auditiva, memória lógica, pronúncia, coordenação motora, atenção e fadiga (Campos, Vilela, Massimi, 2010: Monarcha, 2001. Porém, sem dúvida foi no Instituto de Seleção e Orientação Profissional (ISOP), no Rio de Janeiro, onde foram criados e publicados a maior quantidade de instrumentos psicológicos na década de 50 (Pfromm Netto, 1996).

Um grande incentivador para a área de avaliação psicológica para seleção profissional foi o psicólogo espanhol Emílio Myra y Lopez, que coordenou o ISOP durante 17 anos e ao qual se deve a criação do primeiro periódico cientifico dedicado exclusivamente à Psicologia, conhecido como Arquivos Brasileiro de Psicotécnica, posteriormente denominado Arquivos de Psicologia (Martins, 2014; Rosas, 1995), Juntamente com Lourenço Filho, Myra y Lopez fundou a Sociedade Brasileira de Psicotécnica, conhecida depois como Sociedade Brasileira de Psicologia 
Aplicada, em 1995 (Lourenço, 2000). Deve-se ao ISOP, a origem dos atuais "exames psicotécnicos", iniciados nesta instituição para avaliar motoristas no Rio de Janeiro, envolvendo o teste criado por Myra y Lopez, denominado Psicodiagnóstico Miocinético-PMK (Antunes, 2004).

A década de 1950-1960 foi muito produtiva para os pesquisadores brasileiros interessados na construção de testes para a nossa realidade. Investimentos de agências federais ou estaduais para a seleção e orientação profissional colaboraram, decisivamente, destacando-se o impulso nesta área dado pelo Serviço Nacional de Aprendizagem Industrial (SENAI) em 1942, e Serviço Nacional de Aprendizagem Comercial (SENAC) em 1946 (Pasquali, 2016; Scheeffer, 1970). No campo educacional, pesquisas de desenvolvimento de testes e medidas foram impulsionadas por organizações particulares, como a Sociedade Pestalozzi do Brasil (Campos, 2001) ou por instituições de ensino e pesquisa, tais como o Instituto Nacional de Educação Pública (INEP) (Pfromm Netto, 1981).

Nesta época, foram criados testes brasileiros na área da inteligência, interesses e aptidões, tais como: Inteligência Verbal de Jacyr Maia, em 1950, Bateria de Aptidões-DAT, em 1953, Inteligência Não-Verbal -INV de Pierre Weill em 1955, Inteligência Geral - AG3 do SENAI (Santos, 2003). Algumas baterias de aptidões continuaram a ser utilizadas por várias décadas sem nenhuma preocupação com atualização de normas, tais como a Bateria Fatorial do SENAC, e a Bateria Fatorial do CEPA. Assim se refletem as tendências próprias deste período com a preocupação pelas diferenças individuais, motivação, aptidão e vocação, sobretudo relacionadas à orientação educacional/profissional e à valorização dos testes psicológicos (Antunes, 2002)

O ano de 1962 pode ser considerado como um dos períodos de maior impacto na Psicologia brasileira com a regularização legal da profissão de psicólogos (Lei n ${ }^{\circ}$ $4.119 / 62$ ), na qual o uso de testes psicológicos foi declarado privativo do psicólogo (Decreto No.53.464/64). Também nesta época passou a ser exigido pelo Conselho Nacional de Trânsito (COTRAN) a obrigatoriedade do "exame psicotécnico", assim denominado para todas as pessoas que requisitassem uma carteira de motorista, o que já tinha sido proposto pelo ISOP em 1951.Embora tenha existido um veto presidencial em 1997, extinguindo este exame da avaliação de motoristas, houve uma grande mobilização dos psicológicos e este veto foi derrubado posteriormente, mantendo-se esta exigência até os dias de hoje (Conselho Federal de Psicologia, 2010).

Devem também ser destacados, nesta história, os trabalhos de duas psicólogas, em diferentes pontos do país. Em São Paulo, a professora Odette Lourenção Van Kolck organizou a primeira compilação dos testes disponíveis no país e no exterior em dois volumes, resultante da sua tese de livre docência na USP (Alves, 2004), trazendo os termos de validade e precisão para discutir os testes psicológicos, como também realizou várias publicações na área dos testes projetivos gráficos (Van Kolck, 1968, 1974, 1984): Já no Rio Grande do Sul, Jurema Alcides Cunha, organizou uma série de livros básicos para a formação do psicólogo na área do psicodiagnóstico (Cunha, 2003) tendo também sido responsável pela padronização do Inventário de Inteligência Não Verbal de Pierre Weil, do teste Hotltzman Inkblot Technique (Gomes, 2009), e pela validação da Escala de Depressão de Beck para o país (Cunha, 2001, Cunha 2006)

\section{A organização do movimento de avaliação psicológica}

O percurso histórico da área de avaliação psicológica no país demonstra que este pode ser descrito em várias fases ou ondas, sendo que na primeira houve um grande interesse pelos testes, na segunda uma grande crise ou descrédito, e na terceira, a mais atual, surge um grande desenvolvimento da área (Pasquali, 2010; Wechsler et al., 2014). Assim sendo, o primeiro período de interesse e valorização dos testes psicológicos, como descrito anteriormente, foi sucedido por uma fase de críticas pelos próprios psicólogos, considerando que os testes não representavam as características do povo brasileiro e, principalmente, tinham origem em outros países sem estudos de sua validade e sem normas para nossa cultura (Oakland, Wechsler, \& Maree, 2013). Esta fase de descrédito durou, aproximadamente, duas décadas (19701990), sendo banalizados o estudo e a prática de testes psicológicos no país (Alchieri \& Cruz, 2003).

A preocupação com a qualidade da avaliação psicológica no país estava também presente nas diretorias dos Conselhos Regionais e Federal de Psicologia. Já nos anos de 1980-81, o Conselho Regional de Psicologia-01, em Brasília, organizou reuniões para definir uma política para atender a área dos testes com a presença de pesquisadores de renome como Aroldo Rodrigues e Carolina Bori (Pasquali, 2016). Em 1996, o Conselho Regional de Psicologia-06, em São Paulo, organizou uma reunião para discutir a necessidade de pesquisas na área da avaliação psicológica, com a presença de André Jacquemin, Luiz Pasquali, Claudio Hutz e Solange Wechsler. Neste encontro surgiu a proposta de um grupo de trabalho, coordenado por Oswaldo de Barros Santos com colaboração de Arrigo Angelini para elaborar um anteprojeto de estatutos para fundar um instituto dedicado à pesquisas em avaliação psicológica, inicialmente denominado Instituto Nacional de Avaliação e Pesquisa em Psicologia (INAP). Posteriormente, o INAP foi denominado de Instituto Brasileiro de Avaliação e Pesquisa em Psicologia (IBAPP) (Registro Civil de Pessoas Juridicas, 1997).

Em reunião da Sociedade Brasileira de Psicologia, em 25 de outubro de 1997, foi fundado oficialmente o IBAPP, tendo por presidente Luiz Pasquali. De acordo com a decisão da Assembléia Geral, em 25 de Outubro de 2001, o nome da instituição foi mudado para Instituto Brasileiro de Avaliação Psicológica (IBAP, 2018a) e 
também sua organização da diretoria tendo Luiz Pasquali (presidente passado), Solange Wechsler (presidente atual), Claudio Hutz (presidente futuro), Cilio Ziviane ( vice-presidente), Denise Bandeira (primeira secretaria), Livia Borges (segunda secretaria), Ricardo Primi (primeiro tesoureiro) e Waldiney Gouveia (segundo tesoureiro). Certamente este foi um período decisivo, marcando o crescimento da área de avaliação psicológica, pois os pesquisadores brasileiros decidiram se organizar e planejar estratégias para a melhoria da qualidade dos testes psicológicos no país (Instituto Brasileiro de Avaliação Psicológica, 2002).

A fundação do IBAP liderou o crescimento da área de avaliação psicológica do ponto de vista científico e profissional, pois até o momento as contribuições tinham sido esparsas e de pouco impacto na formação profissional e, principalmente, na qualidade dos instrumentos psicológicos disponíveis no país. Destaca-se a grande contribuição científica com o lançamento da revista Avaliação Psicológica, durante o I Congresso Brasileiro de Psicologia, Ciência e Profissão (Hutz, 2002). Também foi organizado, nesta época, o I Congresso Nacional de Avaliação Psicológica em Campinas (2001) e publicado o primeiro livro pelo IBAP, denominado "Temas em Avaliação Psicológica" (Primi, 2002). No decorrer do seu lançamento, até os dias de hoje, a revista Avaliação Psicológica se tornou uma importante fonte de divulgação de publicações científicas na área, tanto em nível nacional quanto para os países ibero-americanos por ser de acesso aberto pela base eletrônica de periódicos em Psicologia-PEPSIC, tendo a sua qualidade reconhecida pela classificação de A2 na Qualis- CAPES (Avaliação Psicológica, 2018). Deve também ser destacado que o IBAP já organizou oito congressos nacionais até o momento, em diferentes locais do país, com a presença de mais de 1.000 participantes, sendo apresentado, nestes eventos, um crescente número de pesquisas sobre a adaptação e/ou validação de testes psicológicos, para a nossa realidade (Instituto Brasileiro de Avaliação Psicológica, 2018a)

O crescimento do IBAP ocorreu, de forma significativa, pelos esforços dos diferentes presidentes que se sucederam até os dias de hoje: Ricardo Primi, Ana Paula Noronha, Carlos Nunes, Carolina Reppold, Alessandra Seabra, Lucas Carvalho, Monalisa Muniz (Instituto Brasileiro de Avaliação Psicológica, 2018a). Durante estas gestões houve uma sensível melhoria na formação do psicólogo através da realização de cursos de extensão, como também de especialização em avaliação psicológica, oferecido em diferentes locais do país. Além disto a presença política do IBAP no Fórum de Entidades em Psicologia foi reforçada por estas diretorias, exercendo grande importância para o reconhecimento da área, na medida em que o IBAP passou a ser solicitado a colaborar nos eventos da Psicologia. Ressalta-se, nesta parceria do IBAP com o CFP, a publicação das cartilhas online com orientações sobre avaliação psicológica (Conselho Federal de Psicologia, 2010, 2013a), e a organização do "Ano Temático em Avaliação Psicológica" que ocorreu entre 2011, o qual gerou grande mobilização no país através de 120 eventos organizados pelos Conselhos Regionais de Psicologia, e culminou com um Seminário Nacional em Brasília e a publicação de um livro com textos geradores (Conselho Federal de Psicologia, 2013).

Outra marco na organização da área de avaliação psicológica foi a fundação da Sociedade Brasileira de Rorschach e Métodos projetivos (SBRo), em 1993, por André Jacquemin, juntamente com Latife Yazigi, Sonia Regina Loureiro, Cícero Vaz, Sonia Pasian e Anna Elisa Villemor Amaral. Em 2004, o nome desta entidade foi mudado para Associação Brasileira de Rorchach e Métodos Projetivos (ASBRo), com sede na cidade de Ribeirão Preto. Tendo já organizado 8 congressos, esta associação tem proporcionado inestimáveis oportunidades para troca de experiências e melhoria do conhecimento sobre os métodos projetivos (Associação Brasileira de Rorschach e Métodos Projetivos, 2018).

Também em Minas Gerais, devem ser ressaltados os esforços para a melhoria da área de avaliação psicológica. Desde 1993, quando foi lançado o I Encontro de Avaliação Psicológica, já foram realizados 12 Encontros Mineiros de Avaliação Psicológica. Mais recentemente, em junho de 2015, foi fundada a Sociedade de Avaliação e Mensuração Psicológica de Minas Gerais (SAPSI-MG), tendo na diretoria José Alvaro Lelé e Mary Stela Cheueiri (Sociedade de Avaliação e Mensuração Psicológica, 2018). Demonstra-se assim o crescimento da área não só em nível nacional como também regional, possibilitando o avanço cientifico e profissional da área nos mais diferentes contextos.

\section{A melhoria da qualidade científica dos testes psicológicos no país}

A preocupação dos pesquisadores em Psicologia de oferecer instrumentos com qualidade científica, como forma de reagir às críticas aos testes psicológicos, foi o objetivo principal da criação de "laboratórios de pesquisa em avaliação psicológica”. Diferentemente dos primeiros centros de psicologia aplicada no país, também chamados de "laboratórios", nos séculos XVIII e XIX, que se dedicavam principalmente à aplicação de testes educacionais e psicológicos, como já comentado anteriormente, o interesse principal destes laboratórios de pesquisa foi de realizar estudos sistemáticos visando a construção e/ou adaptação de testes psicológicos para o país, que atendessem aos critérios científicos psicométricos de validade, precisão e normas para a população.

O primeiro que se destacou foi o Laboratório de Pesquisa em Avaliação e Medidas (LABPAM), em 1987, na Universidade de Brasília (Pasquali, 2001). Outros laboratórios começaram a surgir, com a mesma finalidade, 
desde esta época, como por exemplo o Laboratório de Mensuração (LAM), na Universidade Federal do Rio Grande do Sul; o Laboratório Interdepartamental de Técnicas de Exame Psicológico (LITEP), na Universidade de São Paulo; o Laboratório de Avaliação e Medidas Psicológicas (LAMP), na Pontifícia Universidade Católica de Campinas; o Laboratório de Avaliação Psicológica e Educacional (LAbaPE), na Universidade São Francisco e o Laboratório de Estudo, Pesquisa e Avaliação Psicológica (LEPAP), na Pontifícia Universidade Católica de Minas Gerais. Atualmente, podem ser contados mais de 46 laboratórios dedicados a este tema, com crescimento notável a partir do ano 2000 (Instituto Brasileiro de Avaliação Psicológica, 2018b; Primi \& Nunes, 2010b).

Outro grande impulso para a melhoria dos instrumentos psicológicos foi dado pelo apoio do Conselho Federal de Psicologia (CFP) às questões relacionadas com avaliação psicológica. Preocupado com a quantidade de queixas sobre o mau uso das práticas envolvendo o uso de testes, o CFP organizou uma comissão de especialistas denominada inicialmente de Comissão Interinstitucional de Avaliação Psicológica e posteriormente de Comissão Consultiva em Avaliação Psicológica. Esta comissão funciona até os dias de hoje, sendo composta por pesquisadores e profissionais da área, cuja função é discutir e propor diretrizes, normas e resoluções no âmbito da avaliação psicológica. Os membros dessa comissão são renovados a cada gestão do CFP, tendo sido já constituídas dez comissões desde 2002 (Primi \& Nunes, 2010a).

Neste sentido, considerando as sugestões decorrentes do I Fórum Nacional de Avaliação Psicológica em 2000, o CFP elaborou um documento (Resolução 025/2001) no qual visava definir os critérios mínimos que os testes deveriam atender para serem aprovados para uso na prática profissional. Esta resolução foi baseada nos parâmetros de entidades internacionais, como da International Test Commission (2000) e a American Educational Research Association, American Psychology Association, National Council of Measurement (1999). A revisão desta resolução, em 2003, definiu 4 critérios como requisitos mínimos e obrigatórios para os instrumentos psicológicos: 1. apresentação da fundamentação teórica do instrumento com ênfase no construto sendo medido; 2. apresentação de evidências empíricas de validade e precisão para as interpretações propostas; 3 . apresentação de dados empíricos sobre as propriedades psicométricas dos itens do instrumento; 4. apresentação do sistema de correção e interpretação dos escore (Conselho Federal de Psicologia, 2003). Esta resolução foi revista em 2018 com maiores esclarecimentos sobre o prazo (15 anos) no qual os instrumentos deveriam apresentar novas normas de avaliação e 20 anos para novos dados sobre a sua validade e precisão para sua aprovação (Conselho Federal de Psicologia, 2018a).

Outro grande marco na avaliação psicológica foi a criação do Sistema de Avaliação de Testes Psicológicos
(SATEPSI), com a finalidade de apresentar parâmetros para certificar a qualidade de um instrumento psicológico, definindo assim requisitos técnicos mínimos (fundamentação teórica, precisão, validade e normatização) para que um teste fosse aprovado para uso por psicólogos (Nunes \& Primi, 2010; Primi, 2010). O processo de avaliação dos testes passou a ser feito por pares anônimos, de maneira similar ao que ocorre com artigos científicos (Primi \& Nunes, 2010a). Uma grande contribuição do SATEPSI para a melhoria da prática psicológica pode ser atribuída à divulgação pública online dos testes aprovados ou reprovados pela comissão de avaliação psicológica. Desta maneira, os psicólogos começaram a tomar contato com as exigências científicas que devem existir para o uso dos testes psicológicos (Sistema de Avaliação dos Testes Psicológicos, 2018). Devido à qualidade científica e rigorosidade do SATEPSI foi chamada a atenção internacional para este sistema, tendo o mesmo sido descrito em uma seção especial do International Journal of Testing dedicada à avaliação dos sistemas internacionais de revisão de testes (Bartram, 2012; Evers, 2012).

Certamente a grande contribuição dada para a melhoria dos instrumentos psicológicos deve-se ao aumento considerável de pesquisadores interessados em avaliação psicológica. Tal fato pode ser observado ao notarmos o crescimento de grupos de trabalho em avaliação psicológica criados na ANPEPP (Cunha, 2018), em quase três décadas desde a sua organização. $\mathrm{O}$ primeiro grupo de trabalho (GT) na ANPEPP na área da avaliação psicológica foi criado em 1989 e teve o nome de Perspectivas em Avaliação e Diagnóstico em Psicologia. Este grupo se reuniu nos congressos consecutivos da ANPEPP com esta mesma denominação, até que em 2008 o número de pesquisadores exigiu a divisão em dois grupos, e em 2014, devido à quantidade de participantes, houve a necessidade da subdivisão em 5 GTS: Pesquisa em Avaliação Psicológica, Métodos Projetivos nos Contextos da Avaliação Psicológica, Avaliação Cognitiva e Neuropsicológica, Avaliação em Psicologia Positiva e Criatividade e Avaliação Psicológica: Personalidade e Psicopatologia (Nascimento \& Vasconcelos, 2016).

Sem dúvida, a expansão dos GTs exemplifica o crescimento da área da avaliação psicológica no país. A organização desses grupos tem sido um espaço científico e até mesmo político para a área. Sob a perspectiva científica e acadêmica, estes grupos têm se organizado para produzir artigos e livros, dando um grande contribuição para a melhoria da formação e atuação na .área da avaliação psicológica (exemplos, Amaral \& Verlang, 2008; Boruchovitch, Santos, \& Nascimento, 2012; Hutz, 2009; 2010, 2014; Noronha, Santos, \& Sisto, 2006; Nunes \& Primi, 2010; Vazquez \& Hutz, 2018; Nakano, 2018.). Por sua vez, estes grupos têm discutido a política da área e produzido documentos com posicionamentos em questões específicas, que são encaminhados posteriormente para o CFP. 


\section{Desafios e Perspectivas Futuras}

Certamente a avaliação psicológica no país teve um grande desenvolvimento na última década. O nível de desenvolvimento alcançado no Brasil não é encontrado, ainda em nenhum outro país da América do Sul, concluindo-se que o país pode servir de modelo para outras nações neste continente (Wechsler at al., 2014). Entretanto, ainda há muito a ser feito para atingir os níveis de países desenvolvidos, no que se refere às características dos instrumentos, à formação de docentes e pesquisadores na área, e a preparação de psicólogos para melhoria dos serviços para a sociedade (Geisinger, 2013).

Quanto à melhoria dos instrumentos, pode-se observar que existem mais testes fazendo uso de análise confirmatória e Teoria de Resposta ao Item (TRI) para analisar a qualidade dos seus itens e as evidências de validade interna (Primi, 2004, 2010). Porém testes que utilizam a testagem adaptativa, ou seja, a administração do teste é adequada ao nível de dificuldade do sujeito, ainda são pouco conhecidos ou utilizados no país. Por sua vez, existe necessidade de avanço para os testes projetivos, que possuem peculiaridades diferentes daquelas empregadas nas técnicas psicométricas e de estudos que abordem a validade consequencial de um instrumento, ou seja, se a avaliação psicológica realmente contribui para intervenções mais eficazes com aquele indivíduo (Primi, Muniz, $\&$ Nunes, 2009).

Nota-se também carência de testes direcionados para indivíduos com necessidades especiais (Wechsler \& Nakano, 2016). A grande maioria dos testes disponíveis são coletados com crianças em salas de aula, adolescentes ou adultos "regulares" e pouco se dispõem, até o momento, de instrumentos que permitam a avaliação de indivíduos com deficiências visuais, auditivas ou até mesmo superdotados. Também predominam testes que avaliam a inteligência e a atenção entre os testes cognitivos aprovados pelo SATEPSI necessitando-se assim de investigação de outras áreas do funcionamento mental e da personalidade (Reppold, Serafini, Gurgel, \& Kaiser, 2017).

A formação dos psicólogos para atuar na área de avaliação psicológica é motivo também de grande preocupação (Mendes, Nakano, Silva \& Sampaio, 2013; Noronha et al., 2013). Além da carga horária insuficiente para a abrangência do conteúdo, predominam técnicas e fundamentos teóricos dos testes nas disciplinas de avaliação psicológica, ao passo que outros conteúdos, tais como princípios de elaboração de testes e de psicometria são praticamente deixados sem discussão. A formação continuada poderia resolver os problemas e minimizar os preconceitos ainda existentes nesta área (Borsa, 2016). O nível de especialização para aqueles que vão atuar na área de avaliação psicológica foi solicitado durante muito tempo ao CFP, porém esta especialidade somente foi reconhecida ao final de 2018 (Conselho Federal de Psicologia, 2018b).

Ressalta-se também o crescimento do número de programas de pós-graduação com linhas de pesquisa em avaliação psicológica, fato inconcebível na época dos 1970-1990, quando esta área era tão desvalorizada. Sem dúvida, a melhor formação de pesquisadores trará impactos benéficos para a docência na graduação e, consequentemente, na qualidade de serviços prestados à sociedade, bem como na oferta de instrumentos com qualidade.

Em conclusão, houve consideráveis avanços na área da avaliação psicológica no país nestas duas últimas décadas. Certamente ainda existem grandes desafios, considerando que ainda é bastante limitado o número de testes aprovados para uso do psicólogo. Entretanto, pode-se esperar que o grande impulso que está sendo realizado na formação de novos profissionais e pesquisadores da área poderá trazer consideráveis avanços não só para a produção de novos instrumentos, mas também para a melhoria dos serviços psicológicos para a sociedade. Assim sendo, são bastante promissoras as perspectivas para o desenvolvimento da área de avaliação psicológica em um futuro próximo.

\section{Referências}

Amaral, A. E. V., \& Werlang, B. S. (2008). Atualizações em Métodos Projetivos para Avaliação Psicológica. São Paulo: Casa do Psicólogo.

Angelini, A. L. (1995). Abertura do I Encontro de Técnicas de Exame Psicológico: Ensino, pesquisa e aplicações. Boletim de Psicologia, 45(102), 9-18.

Alchieri, J. C. \& Cruz, R. M. (2003). Avaliação psicológica: Conceito, métodos e instrumentos. São Paulo, SP: Casa do Psicólogo.

Alves, I. C. B. (2004). Odette Lourenção Van Kolck. Avaliação Psicológica, 3(1), 69-71.

American Educational Research Association, American Psychological Association, and National Council on Measurement in Education. (1999). Standards for Educational and Psychological Testing. Washington DC: American Psychological Association

American Educational Research Association, American Psychological Association, and National Council on Measurement in Education (2014). Standards for Educational and Psychological Testing. Washington DC: American Psychological Association

Antunes, M. A. M. (2002). Psicologia e educação em periódicos brasileiros anteriores a 1962. Psicologia Escolar e Educacional, 6(2), 193-200. doi: 10.1590/S1413-8557200200020001

Associação Brasileira de Rorschach e Métodos Projetivos (2018). Histórico. Associação Brasileira de Rorschach e Métodos Projetivos. Recuperado de http://www.asbro.org.br/historico

Associação Nacional de Pesquisa e Pós-graduação em Psicologia, ANPEPP. (2018). ANPEPP- Grupos de trabalho. Recuperado de http://www. anpepp.org.br/grupos-de-trabalho 
Avaliação Psicológica (2018). Avaliação Psicológica. Informação básica. Recuperado de http://pepsic.bvsalud.org/scielo.php?script=sci_ serial\&pid $=1677-0471$

Bartram, D. (2012). Concluding Thoughts on the Internationalization of Test Reviews. International Journal of Testing, 12(2), 195-201. doi: 10.1080/15305058.2011.649869

Byrne, B., Oakland, T., Leong, F. T. L., Van de Vjiver, F. J. R., Hambleton, R. K. Cheung, F., \& Bartram, D. (2009). A Critical Analysis of Cross-Cultural Research and Testing Practices: Implications for Improved Education and Training in Psychology. Training and Education in Professional Psychology, 3(2), 94-105. doi: 10.1037/a0014516

Borsa, J. C. (2016) Considerações sobre a Formação e a Prática em Avaliação Psicológica no Brasil. Temas em Psicologia 24(1), 131-143. doi: 10.9788/TP2016.1-09.

Boruchovitch, E., Santos, A. A. A., \& Nascimento, E. (2012). Avaliação psicológica nos contextos educativos e psicossociais. São Paulo: Casa do Psicólogo.

Campos, R. H. F. (2001). Helena Antipoff: A synthesis of Swiss and Soviet psychology in the context of Brazilian education. History of Psychology, 4(2), 133-158. doi: 10.1037/1093-4510.4.2.133

Campos, R. H. F., Vilela, A. M. J., \& Massimi, M. (2010). Historiography of psychology in Brazil: Pioneer works, recent developments. History of Psychology, 13(3), 250-276. doi: 10.1037/a0020550

Conselho Federal de Psicologia (2003). Resolução CFP 002/2003 - Define e regulamenta o uso, a elaboração e a comercialização de testes psicológicos. Brasília, DF.

Conselho Federal de Psicologia (2010). Avaliação Psicológica: Diretrizes na regulamentação da profissão. Brasília: Conselho Federal de Psicologia.

Conselho Federal de Psicologia (2013). Relatório do Ano Temático de Avaliação Psicológica 2011/2012. Brasília: Conselho Federal de Psicologia.

Conselho Federal de Psicologia (2013a). Cartilha Avaliação Psicológica. Brasília: Conselho Federal de Psicologia.

Conselho Federal de Psicologia (2018a). Resolução n.9/25 abril/2018. Brasília: Conselho Federal de Psicologia.

Conselho Federal de Psicologia (2018b). APAF reconhece avaliação psicológica como Especialidade em Psicologia. Recuperado de https://site.cfp.org. br/apaf-reconhece-avaliacao-psicologica-como-especialidade-em-psicologia/

Cunha, J. A. (2001) Manual da versão em português das Escalas Beck. São Paulo: Casa do Psicólogo.

Cunha, J. A. (2003). Psicodiagnóstico - V. Porto Alegre: ArtMed.

Cunha, J. A. (2006). Jurema Alcides Cunha. Psicologia, Ciência e Profissão, 26(3), 528-529.

Evers, A. (2012). The Internationalization of Test Reviewing: Trends, Differences, and Results. International Journal of Testing, 12(2), $136-156$. doi: 10.1080/15305058.2012.658932

Evers, A., Muñiz, J., Hagemeister, C., Hstmælingen, A., Lindley, P., Sjöberg, A., \& Bartram, D. (2013). Assessing the quality of tests: Revision of the EFPA review model. Psicothema, 25(3), 283-291. doi: 10.7334/psicothema2013.97.

Flanagan, D.P. \& McDonough, E. M. (Eds.) (2018). Contemporary Intellectual Assessment: Theory, tests and issues. New York: The Guilford Press. Geisinger, K. (Ed.). (2013). APA Handbook of Testing and Assessment in Psychology.Washington, DC: American Psychological Association.

Gomes, W. (2004). Avaliação Psicológica no Brasil: Tests de Medeiros e Albuquerque Psychological Evaluation in Brazil: Tests by Medeirose-Albuquerque. Avaliação Psicologica, 3(1), 59-58.

Gomes, W. (2009). A tradição em avaliação psicológica no Rio Grande do Sul: a liderança e a referência de Jurema Alcides Cunha. Em C. S. Hutz (Ed.). Avanços e polêmicas em avaliação psicológica (pp. 7-24). São Paulo: Casa do Psicólogo.

Gregory, R. J. (2015). Psychological testing: History, principles and applications. Sussex: England: Pearson Education Limited.

Hutz, C. S. (2002). Editorial. Avaliação Psicológica, 1(1).

Hutz, C. S. (2009). (Ed.) Avanços em polêmicas em avaliação psicológica. São Paulo: Casa do Psicólogo.

Hutz, C. S. (2010). Avanços em avaliação psicológica e neuropsicológica com crianças. Vol II. São Paulo: Casa do Psicólogo.

Hutz, C. S. (2014). Avaliação em Psicologia Positiva. São Paulo: Hogrefe

Hutz, C. S., \& Primi, R. (2006) Psychological assessment in Brazil: Current trends and challenges. Score, 28(20), 17-18.

Instituto Brasileiro de Avaliação Psicológica (2002). Boletim do IBAP. Ano I (1). Secretaria do IBAP: Porto Alegre.

Instituto Brasileiro de Avaliação Psicológica (2018a). IBAP-Home. Instituto Brasileiro de Avaliação Psicológica. Recuperado de http://www. ibapnet.org.br/index.php

Instituto Brasileiro de Avaliação Psicológica (2018b). Laboratórios de Avaliação Psicológica em Funcionamento no Brasil. Recuperado de http:// www.ibapnet.org.br/index.php?cd $=8 \&$ descricao $=$ laboratorios

International Test Commission (2000). ITC Guidelines on Adapting Tests. Recuperado de http://www.intestcom.org.

International Testing Commission (2018). Guidelines. Recuperado de https://www.intestcom.org/page/5

Kaplan, R. M., \& Sacuzzo, D. P. (2013). Psychological testing: Principles, applications and issues. California: Wadsworth Cengage Learning

Lourenço, E. (2000). Educação inclusiva: Uma contribuição para a história da Psicologia. Psicologia, Ciência e Profissão, 20(1), $24-49$.

Martins, H. V. (2014). Uma história da psicologia em revista: retomando Mira y López. Arquivos Brasileiros de Psicologia, 66(3), 5-19.

Mendes, L. S., Nakano, T. C., Silva, I. B., \& Sampaio, M. J. (2013). Conceitos de Avaliação Psicológica: Conhecimento de Estudantes e Profissionais. Psicologia, Ciência e Profissão, 33(2), 428-445.

Monarcha, C. (2001). Lourenço Filho e a organização da psicologia aplicada à educação. Brasília: INEP/Ministério da Educação.

Monarcha, C. (2008). "Testes ABC": origem e desenvolvimento. Boletim da Academia Paulista de Psicologia, 28(1), 7-17.

Nascimento, E., \& Vasconcelos, A. G. (2016). O percurso da avaliação psicológica nos simpósios da ANPEPP. Avaliação Psicológica, 15(1), 125-128.

Nakano, T. C. (2018). Psicologia positiva aplicada à educação. São Paulo: Vetor.

Nunes, M. L. T. (2010). Técnicas projetivas com crianças. São Paulo: Casa do Psicólogo.

Noronha, A. P, Castro, N. R., Ottati, M. V., C., Barros, M. V. C., \& Santana, P. R. (2013). Conteúdos e Metodologias de Ensino de Avaliação Psicológica: um Estudo com professores. Paideia, 23(54), 129-139. doi: 10.1590/1982-43272354201315

Noronha, A. P, Santos, A. A. A., \& Sisto, F.F. (2006). Facetas do fazer em avaliação psicológica. São Paulo: Vetor.

Noronha, A. P., \& Reppold, C. T. (2010). Considerações sobre a avaliação psicológica no Brasil. Psicologia, Ciência e Profissão, 30 (n/e), $192-201$.

Nunes, C. H. \& Primi, R. (2010). Aspectos técnicos e conceituais da ficha de avaliação dos testes psicológicos. Em: Santos, A. D., Anache, A. A., Villemor-Amaral, A. D., Werlang, B. S. G., Reppold, C. T., Nunes, C. H. S. S., ... \& Primi, R. (Orgs.). Avaliação Psicológica: Diretrizes na regulamentação da profissão. (pp.101-128). Brasília: Conselho Federal de Psicologia.

Oakland, T. (2009). How universal are test development and use? In E. L. Grigorenko (Ed.), Multicultural psychoeducational assessment (pp. 1-40). New York: Springer Publishing Company. 
Oakland, T., Wechsler, S. M., \& Maree, K. (2013). Test use with children across cultures: a view from three countries. In K. F. Geisinger (Ed.), APA Handbook of Testing and Assessment in Psychology: Vol. 3. Testing and Assessment in School Psychology and Education (pp. 231-257). Washington, D. C.: American Psychological Association.

Pasquali, L. (2001). Testes psicológicos: Conceitos, história, tipos e usos. In L. Pasquali (Org). Técnicas de Exame Psicológico-TEP: Fundamentos das Técnicas Psicológicas. vol I (pp 13-51). São Paulo: Casa do Psicólogo.

Pasquali, 1. (2010). Histórico dos instrumentos psicológicos. In L. Pasquali (Ed). Instrumentação psicológica: Fundamentos e Práticas (pp.11-47). Porto Alegre: Artes Médicas.

Pasquali, L (2016). Os testes psicológicos no Brasil. In L. Pasquali (Ed.) TEP-Técnicas de Exame Psicológico: Os fundamentos (2a . Edição), (pp. 201-220). São Paulo: Vetor Editora.

Pfromm Netto, S. (1981). A Psicologia no Brasil. Em M.G. Ferri e S. Moloyama (Eds.). História das ciências no Brasil. Vol.III. (pp. 235-276). São Paulo/Rio de Janeiro: EDUSP/EPU/CNPQ.

Pfromm Netto, S. (1996). Pioneiros da Psicologia Escolar: Mira y López. Psicologia Escolar e Educacional, 1(1), 87-88.

Portugal, F. T. (2010). Psicologia e história no pensamento social de Manuel Bonfim. Estudos e Pesquisas em Psicologia, 10(2), 596-612.

Primi, R. (2002). (Ed.) Temas em avaliação psicológica. Instituto Brasileiro de Avaliação Psicológica. Campinas: Impressão Digital do Brasil

Primi, R. (2004). Avanços na interpretação de escalas com a aplicação da Teoria de Resposta ao Item. Avaliação Psicológica, 3(1), 53-58.

Primi, R. (2010). Avaliação psicológica no Brasil: fundamentos, situação atual e direções para o futuro. Psicologia: Teoria e Pesquisa, 26(n/e), 25-35. doi: 10.1590/S0102-37722010000500003

Primi, R., Muniz, M., \& Nunes, C. H. S. S. (2009). Definições contemporâneas de validade de testes psicológicos. Em C. S. Hutz (Ed.), Avanços e polêmicas em avaliação psicológica (pp. 243-265). São Paulo: Casa do Psicólogo.

Primi, R \& Nunes, C. H. S. S. (2010a). O SATEPSI: propostas de aprimoramento. In Conselho Federal de Psicologia (CFP), (Ed). Avaliação Psicológica: Diretrizes na regulamentação da profissão. (pp. 129-148). Brasília: Conselho Federal de Psicologia.

Primi, R., \& Nunes, C. H. S. (2010b). Psychological Testing \& Assessment in Brazil: Advances in Research and Federal Regulations. Testing International, 24, 4-5.

Rafante, H. C., \& Lopes, S, R. E (2008). Helena Antipoff e a Fazenda do Rosário: a educação pelo trabalho de meninos “excepcionais" na década de 1940. Revista Terapia Ocupacional, 19(3), 144-152.

Registro Civil de Pessoas Jurídicas (1997). Ata da Fundação do Instituto Brasileiro de Avaliação e Pesquisas em Psicologia - IBAPP, n. 190325. Campinas, SP.

Reppold, C., Serafini, A. J., Gurgel, L. G., \& Kaiser, V. (2017). Avaliação de aspectos cognitivos em adultos: análise de manuais de instrumentos aprovados. Avaliação Psicológica, 16(2), 133-144. doi: 10.15689/AP.2017.1602.03

Rosas, P. (1995). Mira y López: 30 anos depois. Rio de Janeiro: Vetor.

Santos, O. B. (2003). Oswaldo de Barros Santos-Homenageado. Psicologia, Ciênciae Profissão, 23(3), 101. doi: 10.1590/S1414-9893200300030001

Sattler, J. M. (2008). Assessment of children: Cognitive Foundation. New York: Jerome Satler.

Sistema de Avaliação dos Testes Psicológicos (2018). SATEPSI. Recuperado de http://satepsi.cfp.org.br/

Scheeffer, R. (1970). Contribuição da Psicologia ao Campo da Educação nos Últimos 20 anos no Brasil. Arquivos Brasileiros de Psicologia Aplicada, 22(2), 15-20.

Sociedade de Avaliação e Mensuração Psicológica de Minas Gerais- SAPSI-MG (2018). Apresentação. Recuperado de http://www.sapsimg.com.br/.

Van Kolck, O. L. (1968). Interpretação psicológica de desenhos: Três estudos. São Paulo: Pioneira.

Van Kolck, O. L. (1974). Técnicas de Exame Psicológico e suas aplicações no Brasil. Volumes I e II. Petrópolis: Editora Vozes.

Van Kolck, O. (1984). Testes projetivos gráficos no diagnóstico infantil. São Paulo; EPU.

Vazques, A. C. \& Hutz, C. S. (2018) (Eds.) Aplicações da psicologia positiva: Trabalho e organizações. São Paulo: Hogrefe.

Wechsler, S. M. (2001) Avaliação psicológica no Brasil: tendências e perspectivas para o novo milênio. Em Conselho Regional de Psicologia (Ed.). A diversidade da avaliação psicológica: considerações teóricas e práticas. (pp.17-24). João Pessoa: Idéia.

Wechsler, S. M. (2013). El movimiento para el desarrollo de los tests Psicológicos en Brasil. Una experiencia pionera. Psiencia-Revista Latinoamericana de Ciencia Psicológica, 5(2) 010-100. doi: 10.5872/psiencia/5.2.101

Wechsler, S. M., Pérez-Solis,M., Ferreira, C., Magno, I., Contini, No., Bluemn, S., Vivas, E., \& Viloria, C. L. (2010). Test Movement in Iberian-Latin American countries. Testing International, 24(7), 7-8.

Wechsler, S. M., Oakland, T., Leon, C., Vivas, E., Almeida, L., Franco, A., Pérez Solis, \& Contini, N. (2014) Test development and use in five Iberian Latin American Countries. International Journal of Psychology, 49(4) 233-239. doi: 10.1002/ijop.12068

Wechsler, S. M. \& Nakano, T. C. (2016). Cognitive assessment of Brazilian children: Past, present perspectives and challenges. International Journal of School and Educational Psychology, 4(4), 215-224. doi:10.1080/21683603.2016.1163654

recebido em março de 2018 aprovado em fevereiro de 2019

\section{Sobre os autores}

Solange Muglia Wechsler é psicóloga, com doutorado na University of Georgia (Athens, USA). Foi presidente do IBAP e faz parte do Comitê Executivo da International Testing Commission (ITC). Docente pesquisadora da PUC-Campinas onde dirige o Laboratório de Avaliação e Medidas Psicológicas.

Claudio Hutz é psicólogo, com doutorado na University of Iowa (EUA). Foi presidente do IBAP e da Associação de Psicologia Positiva $(\mathrm{ABP}+)$. Docente pesquisador da Universidade Federal do Rio Grande do Sul, onde coordenou o programa de graduação e de pós-graduação.

Ricardo Primi é psicólogo, com doutorado na USP. Foi presidente do IBAP e membro do conselho consultivo do CFP em avaliação psicológica e da CAPES. Docente pesquisador da Universidade São Francisco, onde coordena o Laboratório de Avaliação Psicológica e Educacional (Labape). 